\title{
Pilotversuche mit Cannabis: ein rechtliches Experiment
}

\author{
Katja Cupa *
}

Das Betäubungsmittelgesetz soll neu um einen Experimentartikel ergänzt werden, der die rechtliche Grundlage für die Durchführung von Forschungsprojekten mit Cannabis (Pilotversuche) schaffen soll. Der vorliegende Beitrag zeigt auf, dass die gesetzliche Grundlage dafür bereits heute besteht und dass es in Folge dessen für die Durchführung von Pilotversuchen der vorgeschlagenen Änderung nicht bedarf. Es werden überdies Grenzen der heutigen Rechtslage aufgezeigt sowie dargelegt, inwiefern die vorgeschlagene Gesetzesänderung zukünftig zu Auslegungsproblemen führen würde. Aus den genannten Gründen ist eine Nichtannahme der Gesetzesänderung wünschenswert.

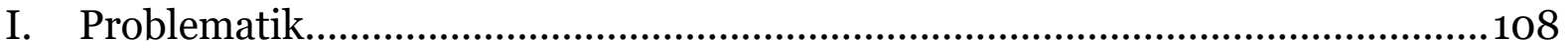

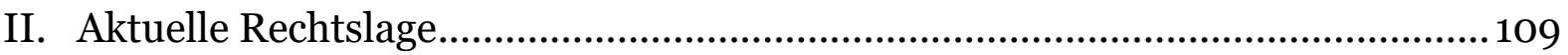

1. Cannabis als verbotenes Betäubungsmittel .............................................. 109

2. Bewilligungspflichtige Handlungen ...................................................... 110

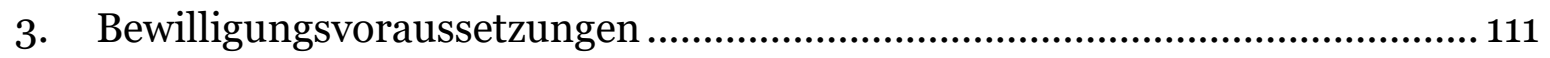

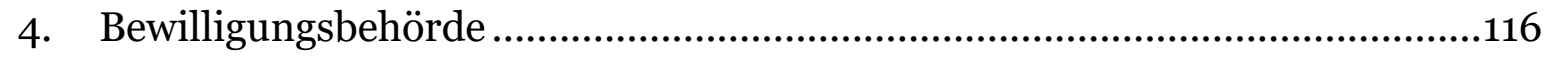

5. Bewilligungsanspruch .................................................................116

III. Abgabe von Cannabis zwecks nicht-medizinischen Konsums......................... 118

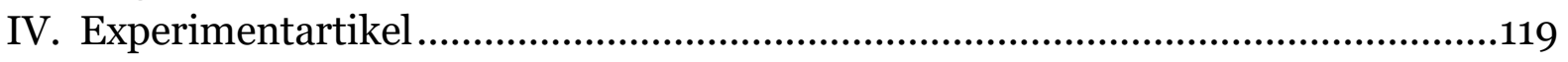

1. Abweichung von Verkehrsverbot und Ausnahmesituationen ........................120

2. Abweichung von Verhaltensvorschriften .............................................. 120

3. Abweichung von Sanktionsnormen .......................................................121

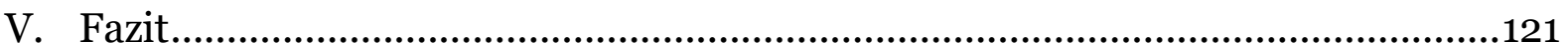

Zitiervorschlag: Katja Cupa, Pilotversuche mit Cannabis: ein rechtliches Experiment, in: sui-generis 2019, S. 107

URL: $\quad$ sui-generis.ch/97

DOI: $\quad$ https://doi.org/10.21257/sg.97

* Katja Cupa, MLaw, Doktorandin an der Universität Zürich, katjamaria.cupa@uzh.ch. 


\section{Problematik}

1 Der Bundesrat eröffnete im Juli 2018 die Vernehmlassung betreffend die Änderung des Betäubungsmittelgesetzes ${ }^{1}$ sowie der dazugehörigen Verordnung. ${ }^{2}$ Im Februar 2019 hat er die Botschaft an das Parlament übergeben. 3 Regulierungsgegenstand sind sogenannte Pilotversuche mit Cannabis, soweit das dabei verwendete Cannabis als Betäubungsmittel qualifiziert wird.4 Der Gesetzesentwurf stellt eine Reaktion auf fünf Motionen dar, welche die Schaffung einer gesetzlichen Grundlage für die Durchführung von Pilotprojekten mit Cannabis verlangen. 5

2 Auslöser für die Einreichung dieser Motionen war der ablehnende Entscheid des Bundesamtes für Gesundheit (BAG) betreffend ein Gesuch der Universität Bern um Ausnahmebewilligung zur Abgabe von Cannabis. ${ }^{6}$ Im Rahmen einer wissen-

1 Bundesgesetz über die Betäubungsmittel und die psychotropen Stoffe vom 3. Oktober 1951 (Betäubungsmittelgesetz, BetmG; SR 812.121).

2 Medienmitteilung des Bundesamts für Gesundheit BAG vom 4. Juli 2018, «Erleichterter Zugang zu Medizinalcannabis und Studien zum Freizeitkonsum».

3 Bundesamt für Gesundheit BAG, Pilotversuche mit Cannabis; Medienmitteilung des Bundesamts für Gesundheit BAG vom 28. Februar 2019, «Pilotversuche mit Cannabis: Botschaft an das Parlament überwiesen»; Botschaft zur Änderung des Betäubungsmittelgesetzes (Pilotversuche mit Cannabis) vom 27. Februar 2019, BBl 20192529 (zit. Botschaft BetmG 2019).

4 Das ist grundsätzlich ab einem THC-Wert von $1 \%$ der Fall (Art. 2 lit. a BetmG i.V.m. Anhang I Verordnung des EDI über die Verzeichnisse der Betäubungsmittel, psychotropen Stoffe, Vorläuferstoffe und Hilfschemikalien vom 30. Mai 2011 [Betäubungsmittelverzeichnisverordnung,

BetmVV-EDI, SR. 812.121.11]).

5 Botschaft BetmG 2019 (Fn. 3), S. 11.

6 Vgl. Begründung der Motionen Experimentierartikel als Grundlage für Studien zur regulierten Cannabis-Abgabe, vom 13. bzw. 14. Dezember 2017: 17.4111 (Sauter), 17.4112 (Barrile), 17.14113 (Rytz), 17.4114 (Bertschy) sowie 17.4210 (Zanetti). schaftlichen Studie sollte untersucht werden, «wie sich ein regulierter Verkauf von Cannabis auf die Konsumentinnen und Konsumenten und auf den illegalen Cannabismarkt in der Stadt Bern auswirkt».7 Das BAG verweigerte gemäss Medienmitteilung die Bewilligung hierfür mit der Begründung, dass die Abgabe von Cannabis zu Genusszwecken gemäss BetmG nicht bewilligungsfähig sei. ${ }^{8}$

3 Das BAG wies in derselben Mitteilung allerdings darauf hin, dass mittels eines Experimentartikels die Möglichkeit zur Durchführung solcher Projekte geschaffen werden könnte. Wissenschaftliche Projekte sollen eine Versachlichung der Diskussionen um Cannabis ermöglichen und dazu beitragen, Optionen für eine künftige Regulierung aufzuzeigen. 9 Aufgrund der Tatsache, dass das Konsumverbot (soweit es besteht) Durchsetzungsschwierigkeiten bereitet, sowie aufgrund der international voranschreitenden Legalisierung ist davon auszugehen, dass das Thema die Politik zukünftig weiter beschäftigen wird. ${ }^{10}$

4 Nachfolgend erörtere ich schwerpunktmässig die aktuelle Rechtslage, um aufzuzeigen, dass kein generelles Konsumverbot für Cannabis besteht und dass die Abgabe von Cannabis im Rahmen wissenschaftlicher Forschung grundsätzlich bewilligungsfähig ist (II.). Dabei mache ich auf Herausforderungen im Zusammenhang mit den Bewilligungsvoraus-

7 Medienmitteilung des Bundesamts für Gesundheit BAG vom 14. November.2017, «Studie der Universität Bern zum Cannabiskonsum nicht bewilligungsfähig» (zit. Medienmitteilung BAG 14. November 2017).

8 Medienmitteilung BAG 14. November 2017 (Fn. 7).

9 Botschaft BetmG 2019 (Fn. 3), S. 5.

10 Botschaft BetmG 2019 (Fn. 3), S. 5. 
setzungen sowie dem Bewilligungsanspruch aufmerksam. Im Anschluss daran vertiefe ich - ebenfalls bezugnehmend auf die bestehenden gesetzlichen Grundlagen - die Abgabe von Cannabis zu nicht-medizinischem Konsum (III.). Im Ergebnis werden diese Erörterungen zeigen, dass zur Durchführung von Pilotprojekten keine Änderung des BetmG notwendig ist. Abschliessend zeige ich anhand von Art. 8a Abs. 2 E-BetmG ${ }^{11}$ beispielhaft auf, dass die Einführung des geplanten Artikels zu Konflikten mit den bestehenden Normen führen würde (IV.).

Art. 8a E-BetmG (Pilotversuche) lautet:

«1 Das Bundesamt für Gesundheit kann nach Anhörung der betroffenen Kantone und Gemeinden wissenschaftliche Pilotversuche mit Betäubungsmitteln des Wirkungstyps Cannabis bewilligen, die:

a. örtlich, zeitlich und sachlich begrenzt sind;

b. es erlauben, Erkenntnisse darüber zu gewinnen, wie sich neue Regelungen auf den Umgang mit diesen Betäubungsmitteln $\mathrm{zu}$ nicht medizinischen Zwecken auswirken; und

c. so durchgeführt werden, dass der Gesundheits- und Jugendschutz, der Schutz der öffentlichen Ordnung sowie die öffentliche Sicherheit gewährleistet sind.

11 Entwurf Bundesgesetz über Betäubungsmittel und psychotrope Stoffe, BBl 20192561 (zit. EBetmG).
2 Der Bundesrat regelt die Voraussetzungen für die Durchführung von Pilotversuchen. Dabei kann er von den Artikeln 8 Absätze 1 Buchstabe d und 5, 11, 13, 19 Absatz 1 Buchstabe f und 20 Absatz 1 Buchstaben d und e abweichen.

3 Betäubungsmittel des Wirkungstyps Cannabis, die im Rahmen der Pilotversuche abgegeben werden, sind von der Tabaksteuer nach Artikel 4 des Tabaksteuergesetzes vom 21. März 1969 befreit.»

6 Die nachfolgende Darstellung ist keine rechtliche Analyse zur Frage der Durchführbarkeit des Forschungsprojekts der Universität Bern. Der Fall eignet sich hier allerdings als Anschauungsbeispiel, weil er zum aktuellen Gesetzesentwurf geführt hat. Es wären mit Blick auf die konkrete Gesuchprüfung weitere rechtliche Überlegungen anzustellen, die hier nicht angesprochen werden können. Es wird überdies keine materielle Analyse des Gesetzesänderungsentwurfs bzw. der dazugehörigen Verordnung vorgenommen, weil sich vorab herausstellt, dass für deren Einführung aus rechtlicher Sicht keine Notwendigkeit besteht.

\section{Aktuelle Rechtslage}

\section{Cannabis als verbotenes Betäubungsmittel}

7 Cannabis wird als sogenanntes verbotenes Betäubungsmittel deklariert (Art. 8 Abs. 1 lit. d BetmG). Es darf demnach grundsätzlich nicht angebaut, eingeführt, hergestellt oder in Verkehr gebracht werden. Der Grund für das Verbot liegt in der Überzeugung des schweizerischen 
Gesetzgebers, dass die medizinische Nutzbarkeit von Cannabis gering ist, das Missbrauchspotenzial und die Schwarzmarktattraktivität jedoch sehr hoch. ${ }^{12}$

Der Begriff «verbotenes Betäubungsmittel» erweist sich bei genauerer Betrachtung als unscharf, denn das Verbot bezieht sich nicht auf Cannabis per se, sondern nur auf dessen Anbau, Einfuhr, Herstellung und Inverkehrbringen. Es ist wichtig zu beachten, dass sich das Verbot von Art. 8 Abs. 1 BetmG nicht auf den Konsum erstreckt. ${ }^{13}$ Zudem gilt das Verbot nicht absolut, sondern kann mittels Bewilligung des BAG überwunden werden, wenn die rechtlichen Voraussetzungen dafür erfüllt sind (Art. 8 Abs. 5 BetmG). Der genaue Umfang des Verbots wird in Kapitel II.2. sowie in III. weiter vertieft.

\section{Bewilligungspflichtige Handlungen}

9 Das genannte Verbot schränkt einerseits die Handlungsmöglichkeiten der Bürger ein und steckt gleichzeitig die Bewilligungskompetenz des BAG ab. ${ }^{14}$ Wer Cannabis anbauen, einführen, herstellen oder in Verkehr bringen will, benötigt dafür nämlich eine Bewilligung (Art. 8 Abs. 5 BetmG). Der Beschrieb der bewilligungspflichtigen Handlungen über-

Botschaft betreffend die Änderung des Bundesgesetzes über die Betäubungsmittel vom 9. Mai 1973, BBl 1973 I 1348 ff. (zit. Botschaft BetmG 1973), 1355; Parlamentarische Initiative Teilrevision des Betäubungsmittelgesetzes, Bericht der Kommission für soziale Sicherheit und Gesundheit des Nationalrates, vom 4. Mai 2006, BBl 20068573 ff. (zit. Bericht SGK-NR 2006), 8608; Thomas Fingerhuth /Stephan Schlegel/Oliver Jucker, Kommentar BetmG, Zürich 2016, Art. 8 N 1.

13 Fingerhuth/Schlegel/Jucker (Fn. 12), Art. 8 N 33; zur Verkehrsunfähigkeit: Gustav Hug-Beeli, Betäubungsmittelgesetz (BetmG), 1. Aufl., Basel 2016, Art. $8 \mathrm{~N} 4 \mathrm{ff}$.

14 Hug-Beeli (Fn. 13), Art. 8 N 51. nimmt darum eine entscheidende Rolle und wird nachfolgend genauer beleuchtet.

10 Der Begriff Anbau wird in der Betäubungsmittelkontrollverordnung 15 einheitlich verwendet im Sinne des Anbaus von Pflanzen und Pilzen (Art. 11 Abs. 2 und Art. 14 BetmKV). Unter Einfuhr wird das Einführen der Ware in das schweizerische Zollgebiet verstanden. ${ }^{16}$ Auffällig ist, dass nur die Einfuhr, nicht jedoch die Ausfuhr erwähnt wird, wo die beiden Begriffe sonst jeweils in einem Atemzug genannt werden. ${ }^{17}$ Die Herstellung wird in Art. 2 lit. c BetmKV definiert, ${ }^{18}$ wogegen das Inverkehrbringen gesetzlich nicht festgelegt ist. Es handelt sich um einen Auffangbegriff, der alle Handlungen, die einem anderen die tatsächliche Verfügungsgewalt am Betäubungsmittel ermöglichen, unter einem Begriff zusammenfasst. Darunter fällt insbesondere auch die Abgabe an den Endverbraucher, ${ }^{19}$ worauf hier wegen ihrer Bedeutung für das Gesuch der Universität Bern besonders hingewiesen wird.

11 Die obige Aufzählung zeigt, dass für verbotene Betäubungsmittel grundsätzlich dieselben Handlungen bewilligungspflichtig sind, wie für nicht verbotene Betäubungsmittel, nämlich der Anbau, die

$15 \overline{\text { Verordnung über die Betäubungsmittelkontrolle }}$ vom 25. Mai 2011 (Betäubungsmittelkontrollverordnung, BetmKV; SR 812.121.1).

16 Ausführlicher dazu Fingerhuth/Schlegel/Jucker (Fn. 12), Art. 19 N 45 oder Hug-Beeli (Fn. 13), Art. $19 \mathrm{~N} 330$.

17 Vgl. Art. 5 BetmG, Art. 7, 23 und 24 BetmKV.

18 «Herstellung: sämtliche Arbeitsgänge vom Gewinnen, Anfertigen, Zubereiten, Be- oder Verarbeiten, Reinigen und Umwandeln bis zum Verpacken, Lagern und Ausliefern des Endprodukts sowie die Qualitätskontrollen und die Freigabe».

19 Hug-Beeli (Fn. 13), Art. 8 N 127 und Art. 19 N 502 ff. 
Herstellung, der Handel, die Abgabe sowie die Ein- (und Aus-)fuhr (Art. 4, 5 und 9 BetmG). Hervorgehoben sei hier wiederum die Bewilligungspflicht für die Abgabe von Betäubungsmitteln. Sie kann leicht überlesen werden, da sie sich aus dem Gesetzestext nur indirekt ergibt: Gemäss Art. 9 BetmG dürfen Medizinalpersonen Betäubungsmittel ohne Bewilligung abgeben. Diese Aussage impliziert, dass Personen, die nicht Medizinalpersonen sind, eine Bewilligung für die Abgabe benötigen. Die besagte Regel wird ebenfalls wiederspiegelt in der BetmKV, wonach für die Abgabe grundsätzlich eine Betriebsbewilligung benötigt wird (Art. 11 BetmKV). ${ }^{20}$

\section{Bewilligungsvoraussetzungen}

12 Wer Cannabis anbauen, einführen, herstellen oder in Verkehr bringen will, benötigt dafür eine Bewilligung. Die Bewilligung kann in materieller Hinsicht erteilt werden, wenn kein internationales Abkommen entgegensteht ${ }^{21}$ und das Betäubungsmittel der wissenschaftlichen Forschung, der Arzneimittelentwicklung oder der beschränkten medizinischen Anwendung dient (Art. 8 Abs. 5 BetmG). Zusätzlich müssen die Bewilligungsvoraussetzungen aus der Betäubungsmittelsuchtverordnung ${ }^{22}$, sowie jene der BetmKV erfüllt sein.

$20 \overline{\text { Vgl. beispielsweise auch Art. 14a BetmG, der die }}$ Bewilligungserteilung für nationale und internationale Organisationen regelt und die Abgabe ebenfalls explizit als bewilligungspflichtige Handlung aufführt.

21 Da bereits Art. 5 BV (Bundesverfassung der Schweizerischen Eidgenossenschaft vom 18. April 1999 [BV; SR 101]) den Völkerrechtsvorrang verankert, gehe ich hier nicht weiter auf diese Voraussetzung ein.

22 Verordnung über Betäubungsmittelsucht und andere suchtbedingte Störungen vom 25. Mai 2011 (Betäubungsmittelsuchtverordnung, BetmSV; SR 812.121.6).

\section{a) Wissenschaftliche Forschung}

13 Da es beim vorgeschlagenen Experimentartikel um die Durchführung wissenschaftlicher Studien geht, wird nur dieser Ausnahmefall beleuchtet. Die Verfügbarkeit von Betäubungsmitteln für Forschungszwecke entspricht einem allgemeinen betäubungsmittelrechtlichen Grundsatz. Cannabis wurde von Anbeginn seiner Regulierung in die Liste der verbotenen Substanzen aufgenommen (Art. 8 aBetmG 1951) und sollte bereits damals für wissenschaftliche Forschung zur Verfügung stehen (Art. 8 Abs. 5 aBetmG 1951).23 Mit der Betäubungsmittelrevision 1973 wurde dieser Grundsatz insofern gestärkt, als dass der Bund «die wissenschaftliche Erforschung der Wirkungsweise der Betäubungsmittel und der Ursachen und Auswirkungen des Betäubungsmittelmissbrauchs sowie die wissenschaftliche Forschung zur Bekämpfung des Betäubungsmittelmissbrauchs fördern» sollte.24 Damit Bundesbeiträge für die Förderung der Forschung zur Verfügung gestellt werden konnten, wurde 1973 Art. 15c aBetmG neu eingefügt. ${ }^{25}$ Der Gesetzgeber bestätigte in der Revision 2006 die damalige Regelung, indem er Art. 15c aBetmG 1973 in seine heutige Form von Art. 3j BetmG mit dem Hinweis überführte, dass sich beide Artikel inhaltlich entsprächen und nach wie vor an der Förderung der Forschung festgehalten werde. ${ }^{26}$

23 Botschaft über die Revision des Bundesgesetzes betreffend Betäubungsmittel vom 9. April 1951, BBl 1951 I 829 ff. (zit. Botschaft BetmG 1951), 855 .

24 Botschaft BetmG 1973 (Fn. 12), 1348.

25 Botschaft BetmG 1973 (Fn. 12), 1351, 1366.

26 Bericht SGK-NR 2006 (Fn. 12), 8605. 
14 Es bleibt zu klären, was unter wissenschaftlicher Forschung zu verstehen ist. Weder das BetmG noch die dazugehörigen Verordnungen enthalten eine entsprechende Definition. Das BetmG verweist jedoch in Art. 3j, der die Förderung der wissenschaftlichen Forschung behandelt, auf das Bundesgesetz über die Förderung der Forschung und der Innovation $^{27}$. Dieses definiert wissenschaftliche Forschung als «methodengeleitete Suche nach neuen Erkenntnissen»; darunter fallen die Grundlagenforschung, deren Ziel der Erkenntnisgewinn ist, und die anwendungsorientierte Forschung, welche Beiträge für praxisbezogene Problemlösungen $\mathrm{zu}$ leisten beabsichtigt (Art. 2 lit. a FIFG). Für die Beantwortung der Frage, was unter wissenschaftlicher Forschung i.S.d. BetmG zu verstehen ist, kann aufgrund des gesetzesexternen Verweises direkt auf die genannte Begriffsdefinition im FIFG abgestellt werden. ${ }^{28}$ Inhaltlich gibt Art. 3j BetmG eine nicht abschliessende Liste von möglichen Forschungsbereichen an, die sich in ähnlicher Form in der Botschaft BetmG 1973 wiederfindet. ${ }^{29}$ Da es sich um eine beispielhafte Aufzählung handelt, lässt sich daraus keine Einschränkung des Forschungsbegriffs ableiten. Die BetmSV, welche gemäss ihren Erläuterungen die Anforderungen an die Ausnahmebewilligung zu präzisieren beabsichtigt, ${ }^{30}$ fügt

27 Bundesgesetz über die Förderung der Forschung und der Innovation vom 14. Dezember 2012 (FIFG; SR 420.1).

28 Hug-Beeli (Fn. 13), Art. 3j N 2.

29 Art. 3j BetmG: « [...] a. Wirkungsweise abhängigkeitserzeugender Stoffe; b. Ursachen und Auswirkungen suchtbedingter Störungen; c. präventive und therapeutische Massnahmen; d. Verhinderung oder Verminderung suchtbedingter Störungen; e. Wirksamkeit von Wiedereingliederungsmassnahmen.»

30 Erläuterungen des BAG zur Verordnung über die Betäubungsmittelkontrolle (Betäubungsmittel- dieser Definition der wissenschaftlichen Forschung nichts hinzu. In materieller Hinsicht ist ein Gesuch um Abgabe von Cannabis demnach bewilligungsfähig, wenn damit Forschung i.S.d. FIFG betrieben wird.

15 Ergänzend dazu verlangt die BetmSV, dass der Nachweis für die Voraussetzungen der Guten Laborpraxis erbracht wird (Art. 28 Abs. 1 lit. b und Abs. 2 lit. b BetmSV). Letztere wird separat in der Verordnung über die Gute Laborpraxis ${ }^{1}$ geregelt und gilt für «nichtklinische Prüfungen von Stoffen, Zubereitungen und Gegenständen» (Art. 2 GLPV). Dieser Nachweis ist eine formale Anforderung, die objektiv nur dann erbracht - und damit auch nur dann verlangt - werden kann, wenn das Forschungsprojekt so aufgesetzt ist, dass es in den Anwendungsbereich der GLPV fällt (wenn bspw. im Labor geforscht wird). ${ }^{2}$ Bisweilen wird aus dem Verordnungstext der Rückschluss gezogen, dass nur Laborforschung zulässig sei.33 Diese Interpretation ist vor dem Hintergrund der hinlänglich definierten gesetzlichen Grundlage

kontrollverordnung, BetmKV) und zur Verordnung über die suchtbedingten Störungen (Betäubungsmittelsuchtverordnung, BetmSV), (zit. Erläuterungen BetmKV/BetmSV), S. 34 .

${ }_{31}$ Verordnung über die Gute Laborpraxis vom 18. Mai 2005 (GLPV; SR 813.112.1).

32 Bericht SGK-NR 2006 (Fn. 12), 8606.

33 Siehe dazu beispielsweise Hug-Beeli (Fn. 13), Art 8 N 68 und Christian Huber, Die gesetzliche Grundlage einer kontrollierten Heroinabgabe, SJZ 88/1993, S. 47 ff., S. 48: Beide sind der Auffassung, dass der Gesetzgeber mit Forschung nur Laborforschung meinte. Die angegebenen Fundstellen zum Beleg dieser Aussage beinhalten jedoch keinen Hinweis auf Laborforschung. Auch im Bericht des Bundesrats in Erfüllung der Motion 14.4164, Kessler, vom 11 Dezember 2014, S. 21, heisst es, der Forschungsbegriff orientiere sich an der Laborforschung, ebenfalls ohne Beleg oder Begründung. 
der wissenschaftlichen Forschung nicht nachvollziehbar.

Des Weiteren verlangt die BetmSV, dass nicht nur Personen, die Cannabis anbauen, einführen, herstellen oder in Verkehr bringen wollen, eine Ausnahmebewilligung benötigen, sondern überdies auch Personen, die damit Forschung zu betreiben beabsichtigen (Art. 28 Abs. 1 lit. b BetmSV). Diese Vorschrift überzeugt aus verschiedenen Gründen nicht: Erstens erklärt das BetmG das wissenschaftliche Forschen nicht als bewilligungspflichtige Handlung. Aufgrund der fehlenden Bewilligungspflicht existiert auch die entsprechende Bewilligungskompetenz des BAG nicht. Zweitens handelt es sich bei der BetmSV um eine Vollzugsverordnung, welche allein der Konkretisierung des BetmG dienen soll.34 Das Erfordernis einer Ausnahmebewilligung für Forschungseinrichtungen ist im Gesetz nicht vorgesehen. Eine entsprechende Delegationsnorm, welche einen Ausbau der bewilligungspflichtigen Handlungen auf Verordnungsstufe zuliesse, ist im BetmG nicht vorhanden und wäre auf seine Vereinbarkeit mit dem Erfordernis der genügenden gesetzlichen Grundlage wichtiger rechtssetzender Bestimmungen i.S.v. Art. 164 BV zu prüfen. 35 Drittens ist eine solche Bewilligung überflüssig, denn sofern eine Forschungseinrichtung im Rahmen ihres Forschungsprojekts mit Cannabis verkehrt, wird sie als Folge dessen eine ent-

35 Vgl. zum Unterschied zwischen Vollzugsverordnung und gesetzesergänzender Verordnung: Peter Hettich, in: Giovanni Biaggini/Isabelle Häner/Urs Saxer/Markus Schott, Fachbuch Verwaltungsrecht: Expertenwissen für die Praxis, Zürich 2015, S. 829. sprechende Anbau- oder Betriebsbewilligung beantragen müssen.

17 Die BetmSV nennt zuletzt die Nachweise, welche bei einem Bewilligungsgesuch für Anbau, Einfuhr, Herstellung und Inverkehrbringen $\mathrm{zu}$ erbringen sind. Dabei handelt es sich um Formalitäten, wie Angaben über die gesuchstellende Person, Verwendungszweck der Betäubungsmittel sowie Menge und Bezugsort derselben (Art. 28 Abs. 2 lit. a BetmSV).

\section{b) Allgemeine Bewilligungs- voraussetzungen}

18 Neben der materiellen Voraussetzung der wissenschaftlichen Forschung und den eben genannten Formalitäten, müssen für den Anbau von Cannabis die Voraussetzungen der Anbaubewilligung (Art. 11 Abs. 2 i.V.m. Art. 14 BetmKV), für die Herstellung und das Inverkehrbringen die Voraussetzungen der Betriebsbewilligung (Art. 11 Abs. 1 i.V.m. Art. 12 BetmKV) und für die Einfuhr die Voraussetzungen der Einfuhrbewilligung (Art. 23 i.V.m. Art. 24 BetmKV) erfüllt werden. Die Voraussetzungen sind in der BetmKV abschliessend geregelt und unstrittig, weswegen sich eine Wiederholung an dieser Stelle erübrigt. Unterschiedlich können allerdings die Gründe sein, die zur Anwendung der eben genannten Bewilligungsvoraussetzungen führen, so dass es interessant scheint, diese hier zu diskutieren.

19 Hinsichtlich Anbau- und Betriebsbewilligung wird in der Literatur bislang die Auffassung vertreten, dass deren Voraussetzungen für sogenannte verbotene Betäubungsmittel grundsätzlich nicht gel- 
ten. ${ }^{36}$ Art. 4 Abs. 1 BetmG zeige deutlich, dass es sich bei der Bewilligung für Betäubungsmittel im Allgemeinen und jener für verbotene Betäubungsmittel um zwei unterschiedliche Bewilligungen handle; trotzdem seien die Voraussetzungen aus der BetmKV, die unbestrittenermassen für nicht verbotene Betäubungsmittel gelten, auch auf die verbotenen Betäubungsmittel anwendbar, da die Anforderungen bei verbotenen Substanzen nicht geringer sein könnten als bei nicht verbotenen Substanzen. 37 Aufgrund der Terminologie, sowie der Verteilung der Bewilligungsvoraussetzungen auf die BetmKV und die BetmSV, erscheint diese Argumentation auf den ersten Blick nachvollziehbar.

Auf den zweiten Blick ergeben sich jedoch Gründe, die dafür sprechen, dass die Voraussetzungen der Anbau- und Betriebsbewilligung auch für verbotene Betäubungsmittel anwendbar sind, nicht nur aus einer Plausibilitätskontrolle, sondern aus dem Gesetz bzw. aus der BetmKV selbst: Es wurde bereits aufgezeigt, dass die bewilligungspflichtigen Handlungen für alle Betäubungsmittel (verbotene sowie nicht verbotene) deckungsgleich sind. Art. 11 BetmKV, der die Anbau- und Betriebsbewilligung definiert, knüpft einzig an den bewilligungspflichtigen Handlungen an. Er bezieht sich dabei nicht auf die Unterscheidung zwischen verbotenen oder nicht verbotenen Betäubungsmitteln, ebenso wenig wie auf die Begriffe Bewilligung oder Ausnahmebewilligung, und statuiert überdies auch keinen Vorbehalt für ver-

36 Fingerhuth/Schlegel/Jucker (Fn. 12), Art. 8 N 34; Hug-Beeli (Fn. 13), Art. $8 \mathrm{~N} 51 \mathrm{ff}$.

37 Fingerhuth/Schlegel/Jucker (Fn. 12), Art. 8 N 34; weniger detailliert: Hug-Beeli (Fn. 13), Art. 8 N 56 und 58. botene Betäubungsmittel nach Art. 8 BetmG. Die Bestimmung in Art. 11 BetmKV hat folgenden Wortlaut:

«1Wer kontrollierte Substanzen, ausgenommen Hilfschemikalien, herstellen, beziehen, vermitteln, einund ausführen, abgeben oder mit diesen Handel treiben will, benötigt vorgängig eine Betriebsbewilligung.

${ }^{2}$ Wer Pflanzen oder Pilze, die kontrollierte Substanzen enthalten, anbauen will, benötigt eine Anbaubewilligung.»

21 Die in Art. 12 und 14 BetmKV für Anbauund Betriebsbewilligungen genannten Voraussetzungen gelten gemäss Verordnungstext uneingeschränkt für «kontrollierte Substanzen», also auch für verbotene Betäubungsmittel wie Cannabis. Damit ergibt sich direkt aus dem Verordnungstext, dass die Bewilligungsvoraussetzungen für die Anbau- und Betriebsbewilligung für alle Betäubungsmittel gelten. Auf den Gesetzestext abstellend (Bewilligung und Ausnahmebewilligung), ist die Nichtanwendung der genannten Voraussetzungen aus der BetmKV auf verbotene Betäubungsmittel demnach nicht gerechtfertigt. Dies erscheint insofern auch sinnvoll, als dass für alle Betäubungsmittel dieselben Handlungen bewilligungspflichtig sind.

Diesem Verständnis könnte entgegengehalten werden, Art. 11 BetmKV dürfe ausschliesslich in Kombination mit Art. 5 BetmKV gelesen werden, weil dieser mit der Überschrift Betriebsbewilligung versehen ist und sich nicht zu verbotenen Betäubungsmitteln äussert. Dieses Argument überzeugt aus zwei Gründen 
nicht. Einerseits gibt es eine klare systematische Trennung zwischen Art. 5 BetmKV, der die Bewilligungsbehörde für nicht verbotene Betäubungsmittel konkretisiert, und Art. 11 BetmKV, der die Bewilligungsvoraussetzungen für die bewilligungspflichtigen Handlungen bekannt gibt. Der Begriff Betriebsbewilligung ist gemäss Art. 11 BetmKV gerade durch die bewilligungspflichtigen Handlungen definiert, nicht durch die behördliche Zuständigkeit. Andererseits äussert sich Art. 5 BetmKV zwar nicht zur Bewilligungserteilung für verbotene Betäubungsmittel, er spricht sich aber auch nicht über die Bewilligungskompetenz für die Anbaubewilligung aus. Wollte man die Definition von Anbau- und Betriebsbewilligung an Art. 5 BetmKV anstatt an Art. 11 BetmKV festmachen, bliebe die Anbaubewilligung undefiniert und es gäbe keine bewilligungsfähige Behörde.

Der einen sowie der anderen Argumentationslinie folgend, gelangt man zum selben Ergebnis, nämlich zur Anwendung der Bewilligungsvoraussetzungen aus der BetmKV auf verbotene Betäubungsmittel. Restlos überzeugend wirkt keine der beiden Begründungen.

24 Die hier vorgestellte Lesart führt jedoch einen Schritt weiter zur Anwendung von Art. 16 BetmKV, der die Wirkung der Betriebsbewilligung regelt. Die Folge der Bewilligungen wurde bislang nicht diskutiert, weil es offensichtlich schien, dass die Bewilligung im Einzelfall die Zulässigkeit der bewilligungspflichtigen Handlungen erklärt. Der Verordnungstext ist diesbezüglich aber nicht so eindeutig wie erwartet. Gemäss Art. 16 BetmKV ist der Inhaber einer Betriebsbewilligung zur
Abgabe und zur Vermittlung kontrollierter Substanzen an Personen berechtigt, die entweder selbst eine Bewilligung besitzen oder von der Bewilligungspflicht befreit sind. Damit regelt der Artikel entgegen seiner Überschrift - nicht die Wirkungen, sondern höchstens einen Teil der Wirkungen, nämlich bezüglich Abgabe und Vermittlung. Wollte die Norm die vollständigen Wirkungen benennen, müsste sie sich gemäss Art. 11 BetmKV auch über die Zulässigkeit des Herstellens, Beziehens, Vermittelns, Abgebens (an Konsumenten) oder des Handels äussern. Auslegungsbedürftig ist beim aktuellen Wortlaut insbesondere der Begriff Abgabe. Denn gemäss Art. 16 BetmKV bezeichnet die Abgabe die Übermittlung an diejenige Person, welche die Abgabe an den Endverbraucher potentiell vornehmen wird und erwähnt die Abgabe an den Konsumenten selbst, für die gemäss Art. 8 Abs. 5 BetmG i.V.m. Art. 11 BetmKV eine Bewilligung erteilt werden kann, gerade nicht.

25 In Bezug auf die Einfuhrbewilligung wird bislang die Meinung vertreten, dass neben der Ausnahmebewilligung des BAG noch eine Einfuhrbewilligung des Schweizerischen Heilmittelinstituts notwendig sei.38 Gleiches ist den Erläuterungen $\mathrm{zu}$ den Verordnungen zu entnehmen.39 Dazu folgende kritische Überlegungen: Erstens ist es nicht einleuchtend, wieso zwei Behörden dieselbe Handlung bewilligen sollen. Zuerst verfügt das BAG und danach das Schweizerische Heilmittelinstitut über die Einfuhr? Zweitens enthält Art. 5 BetmG, der

${ }_{38} \overline{\text { Vgl. beispielsweise Fingerhuth/Schlegel/Jucker }}$ (Fn. 12), Art. 8 N 34; Hug-Beeli (Fn. 13), Art. 8 N 60.

39 Erläuterungen BetmKV/BetmSV (Fn. 30), S. 34. 
die Ein- und Ausfuhr regelt, keinen Vorbehalt zu Gunsten von Art. 8 BetmG. Es gilt also vorbehaltslos, dass Ein- und Ausfuhr von Betäubungsmitteln einer Bewilligung des Schweizerischen Heilmittelinstituts bedürfen. Drittens verlangt Art. 24 Abs. 1 lit. c BetmKV als Voraussetzung zur Bewilligungserteilung, dass eine gültige Bewilligung zum Verkehr besteht, wie beispielsweise eine Bewilligung nach Art. 8 Abs. 5 BetmG. Dadurch wird sichergestellt, dass die notwendige materielle Prüfung vorgängig durch das BAG stattgefunden hat. Viertens haben wir bei den bewilligungspflichtigen Handlungen (II.2.) gesehen, dass Art. 8 Abs. 5 BetmG nur von Einfuhr und nicht von Ein- und Ausfuhr spricht. Vor dem hier genannten Hintergrund ist es denkbar, dass es sich dabei um ein redaktionelles Versehen handelt - aus gesetzessystematischer Sicht hätte die Einfuhr nicht erwähnt werden sollen.

Im Ergebnis würde die hier vorgestellte Lesart bedeuten, dass gemäss Art. 5 BetmG i.V.m. Art. 24 Abs. 1 lit. c BetmKV die Ein- und Ausfuhr von Cannabis bewilligungspflichtig ist und dass die Bewilligung vom Schweizerischen Heilmittelinstitut erteilt wird, sofern der Antragsteller über eine Anbau- oder Betriebsbewilligung des BAG verfügt, die für eine Ausnahmesituation i.S.v. Art. 8 Abs. 5 BetmG erteilt wurde. Zuletzt sei in diesem Zusammenhang Art. 11 Abs. 1 BetmKV erwähnt, demzufolge die Einund die Ausfuhr vorgängig einer Betriebsbewilligung bedürfen. Diese Aussage scheint deklaratorischer Natur zu sein. Denn eine Betriebsbewilligung ermächtigt nicht zur Ein- und Ausfuhr sondern ist Voraussetzung für die eigentliche Einund Ausfuhrbewilligung. Es wäre logi- scherweise nicht notwendig, diese beiden Handlungen in Art. 11 BetmKV aufzuführen.

27 Schliesslich kann die Ausnahmebewilligung für wissenschaftliche Forschung nach Art. 8 Abs. 5 BetmG nichts anderes sein als eine Anbau- oder Betriebsbewilligung, die vom BAG nur bei Vorliegen der genannten Ausnahmesituation erteilt werden kann. Die obige Diskussion vermochte weder alle Fragen, noch alle möglichen Antworten darzulegen, die sich im heutigen System von Bewilligungspflicht, -voraussetzung und -wirkung ergeben. Sie zeigt aber, dass die aktuelle Rechtslage systematische wie auch sprachliche Unstimmigkeiten in sich birgt, die für den Rechtssuchenden eine Herausforderung darstellen. Als Folge davon dürfte die Rechtsunsicherheit entsprechend gross sein.

\section{Bewilligungsbehörde}

28 Nach der oben vorgestellten Lesart wird die Anbau- und Betriebsbewilligung für verbotene Betäubungsmittel vom BAG erteilt (Art. 8 Abs. 5 BetmG und Art. 8 BetmKV) und die Ein- und Ausfuhrbewilligung für verbotene Betäubungsmittel vom Schweizerischen Heilmittelinstitut.

\section{Bewilligungsanspruch}

29 Während für Betäubungsmittel eine $B e$ willigung verlangt wird (Art. 4, 5 und 9 BetmG), wird für verbotene Betäubungsmittel eine sogenannte Ausnahmebewilligung verlangt (Art. 8 Abs. 5 BetmG). Diese Begriffe können irreführend sein. Denn bei einer Bewilligung, ebenso wie bei einer Ausnahmebewilligung, formuliert das Gesetz ein Verbot 
mit einem Bewilligungsvorbehalt. 40 In beiden Fällen handelt es sich also um Ausdruck ein und derselben Handlungsform der Verwaltung, nämlich den Erlass einer Verfügung i.S.v. Art. 5 VwVG41.42

Der Begriff Ausnahmebewilligung ist im BetmG nicht definiert. Es handelt sich dabei um einen Begriff aus der Lehre, der eine Situation beschreibt, in welcher «von der im Normalfall geltenden Regelung insbesondere von einer bestimmten polizeilichen Vorschrift - in einzelnen Sonderfällen gestützt auf eine gesetzliche Ermächtigung abgewichen werden darf».43 Er stelle ein Mittel zur Vermeidung von Härtefällen dar. Da der Gesetzgeber nicht alle Einzelfälle regeln könne, sei die Ausnahmebewilligung dort ein geeignetes Instrument, wo aus überwiegenden privaten oder öffentlichen Interessen eine Ausnahme vom Verbot zur Wahrung des Verhältnismässigkeitsprinzips angezeigt sei. 44 Hauptsächlich geht es bei der Unterscheidung zwischen Bewilligung (auch Polizeierlaubnis) und Ausnahmebewilligung darum, den behördlichen Ermessensspielraum, der bei der Ausgestaltung der Ausnahmebewilligung besteht, zu rechtfertigen, 45 denn das pflichtgemässe Ermessen unterliegt im Allgemeinen nicht der richterlichen Kontrolle, 46 was die Durchsetzung des Rechtsanspruchs

40 Hettich (Fn. 35), S. 827.

41 Bundesgesetz über das Verwaltungsverfahren vom 20. Dezember 1968 (Verwaltungsverfahrensgesetz, VwVG; SR 172.021).

42 Ulrich Häfelin/Georg Müller/Felix Uhlmann, Allgemeines Verwaltungsrecht, 7. Aufl., Zürich 2016, N 895, N 2650 ff.; Hettich (Fn. 35), S. 827.

43 Häfelin/Müller /Uhlmann (Fn. 42), N 2665.

44 Hettich (Fn. 35), S. 827.

45 René Wiederkehr/Paul Richli, Praxis des Allgemeinen Verwaltungsrechts - Band II: Eine systematische Analyse der Rechtsprechung, Bern 2014, S. 228.

46 Häfelin/Müller/Uhlmann (Fn. 42), N 2675. auf Erteilung einer Bewilligung erschweren kann.

31 Ob im konkreten Fall eine Ausnahmebewilligung im eben beschriebenen Sinn vorliegt, entscheidet sich nach Massgabe der materiellen rechtlichen Grundlage, nicht nach der Bezeichnung.47 Daraus folgt, dass aus der Bezeichnung als Ausnahmebewilligung nicht automatisch der Rückschluss gezogen werden kann, dass selbst bei Vorliegen der Bewilligungsvoraussetzungen kein Rechtsanspruch auf Erteilung der Bewilligung bestünde.

32 Im vorliegenden Fall verlangt Art. 8 Abs. 5 BetmG eine (Ausnahme-)Bewilligung. Gesetz und Verordnungen etablieren eine zwei-stufige Prüfung: Erstens ist zu prüfen, ob die materiellen Voraussetzungen aus Art. 8 Abs. 5 BetmG mit den entsprechenden Nachweisen gemäss Art. 28 Abs. 2 BetmSV (sofern massgeblich) erfüllt sind. Diese Bewilligungsvoraussetzungen sind hinlänglich bestimmt: In materieller Hinsicht ist die wissenschaftliche Forschung durch den Bezug auf das FIFG definiert und die Nachweise nach Art. 28 BetmSV sind nicht interpretationsbedürftig. Zweitens ist zu prüfen, ob die allgemeinen Voraussetzungen für eine Anbau- bzw. Betriebsbewilligung gemäss BetmKV erfüllt sind. Die dort abschliessend aufgeführten Voraussetzungen sind ebenfalls hinlänglich bestimmt. Das bedeutet, dass die rechtlichen Grundlagen zur Regelung der Ausnahmesituation der wissenschaftlichen Forschung, basierend auf welchen eine Verfügung erlassen werden kann, keinen

47 Markus Müller, in: Christoph Auer/Markus Müller/Benjamin Schindler, VwVG: Kommentar zum Bundesgesetz über das Verwaltungsverfahren, 2. Aufl., Zürich 2018, S. 68. 
Ermessensspielraum formulieren. Sofern die Voraussetzungen erfüllt sind, besteht folglich ein Bewilligungsanspruch. $4^{8}$

\section{Abgabe von Cannabis zwecks nicht- medizinischen Konsums}

33 Das Gesuch der Universität Bern um Erteilung einer (Ausnahme-)Bewilligung für die Abgabe von Cannabis wurde vom BAG gemäss eigenen Angaben mit der Begründung abgelehnt, dass der Konsum von Cannabis zu Genusszwecken verboten bleibe, auch im Rahmen von wissenschaftlichen Studien. 49

34 M.E. ist das Gesuch um Abgabe von Cannabis im Rahmen einer wissenschaftlichen Studie auf der bestehenden gesetzlichen Grundlage jedoch bewilligungsfähig. Die Begründung des BAG vernachlässigt, dass nur der unbefugte Konsum verboten ist (Art. 19a BetmG) und sich die Befugnis eben gerade aus der Ausnahmebewilligung ergibt. Das wird nachfolgend vertieft.

Zuerst gilt es nochmal festzuhalten, dass der Konsum keine bewilligungspflichtige Handlung ist (vgl. II.2.). Als Folge davon kann der Konsum grundsätzlich nicht unbefugt erfolgen und muss darum straffrei bleiben. Gleichwohl besteht weitgehend Einstimmigkeit darüber, dass nur der ärztlich verordnete Konsum befugt sei.5o

48 Zum Rechtsanspruch im Fall von unechten Ausnahmeregeln vgl.: Thomas Heiniger, Der Ausnahmeentscheid: Untersuchungen zu Ausnahmeermächtigung und Ausnahmebewilligung, Diss. Zürich 1985, S. $44 \mathrm{ff}$.

49 Medienmitteilung BAG 14. November 2017 (Fn. 7).

50 Peter Albrecht, Die Strafbestimmungen des Betäubungsmittelgesetzes (Art. 19-28l BetmG), 3. Aufl., Bern 2016, Art. 19a N 2; Finger-
36 Eine mögliche Erklärung dafür ist, dass die Einführung der Strafnorm betreffend den Konsum eines der Ziele der Revision des BetmG 1975 war.51 Während der Konsum bis dahin nicht mit Strafe bedroht war, machten sich Konsumenten indirekt gleichwohl strafbar, weil dem Konsum naturgemäss eine strafbare Handlung - wie beispielsweise der Kauf - vorangehen muss. ${ }^{22}$ Die Tatsache, dass der Konsum eigentlich gerade straffrei war, weckte das Bedürfnis, Konsumenten in strafrechtlicher Hinsicht zu privilegieren; um dieses Ziel zu erreichen, sollte der unbefugte Konsum selbst ein Straftatbestand werden.53 Das erklärt die Akzeptanz (nicht die Rechtmässigkeit) der Bestrafung des Konsums. Es erklärt aber noch nicht, welcher Konsum unbefugt erfolgen soll.

37 Aufgrund fehlender Bewilligungspflicht des Konsums wird das Verständnis dessen, was befugt bzw. unbefugt ist, konstruiert: «Die Erlaubnis zum Konsum leitet sich vielmehr indirekt aus der Befugnis derjenigen Person ab, welche den Stoff an Konsumenten abgeben darf.»54 Wie oben ausgeführt, ist die Abgabe von Cannabis in den Ausnahmefällen von Art. 8 Abs. 5 BetmG bewilligungsfähig. Konsequenterweise erfolgt der Konsum von Cannabis dann befugt, wenn er im

huth/Schlegel/Jucker (Fn. 12), Art. 19a N 9; HugBeeli (Fn. 13), Art. 19a N 253.

51 Botschaft BetmG 1973 (Fn. 12), 1367; weiterführend Peter Albrecht, Die strafrechtliche Beurteilung der Drogenkonsumenten (Bemerkungen zur Auslegung des Art. 19a Ziff. 1 BetmG), BJM 1983, S. $217 \mathrm{ff}$.

52 Botschaft BetmG 1973 (Fn. 12), 1367, mit Verweis auf BGE 95 IV 179.

53 Botschaft BetmG 1973 (Fn. 12), 1368; Albrecht (Fn. 50), Art. 19a N 1, mit weiteren Verweisen; Fingerhuth/Schlegel/Jucker (Fn. 12), Art. 19a N 1.

54 Albrecht (Fn. 5O), Art. 19a N 27. 
Rahmen wissenschaftlicher Forschung nach Art. 8 Abs. 5 BetmG stattfindet. 55

38

ie eingangs erwähnt, erscheint der Standardfall des ärztlich verordneten Konsums von Betäubungsmitteln unbestritten und wird sodann auch regelmässig im Zusammenhang mit der korrespondierenden Strafnorm ausgeführt.56 Dabei können die Anwendungsfälle von Art. 8 Abs. 5 BetmG, die für verbotene Betäubungsmittel bedeutsam sind, leicht in Vergessenheit geraten, gerade weil sie nicht alltäglich auftreten. Gleichwohl geben verallgemeinernde Aussagen, wie der Konsum von Cannabis sei verboten, die Rechtslage unzutreffend wieder.

39 Erwähnenswert ist nicht zuletzt, dass ein wissenschaftliches Projekt zur kontrollierten Abgabe von Heroin, Morphin und Methadon in den 1990er Jahren gestützt auf Art. 8 Abs. 5 BetmG erfolgte. 57 Da es dabei ebenfalls um die Abgabe bzw. den Konsum eines verbotenen Betäubungsmittels zwecks wissenschaftlicher Forschung ging, ist auf den ersten Blick nicht erkennbar, was bezüglich des aktuellen Forschungsprojektes zu einer anderen rechtlichen Beurteilung führen soll. Dies umso weniger, als dass bereits damals - nach hier vertretener Meinung fälschlicherweise - von einem Konsum-

$55 \overline{\text { Albrecht (Fn. 50), Art. 19a N 28, mit explizitem }}$ Bezug auf die Einnahme von verbotenen Betäubungsmitteln im Rahmen eines Forschungsprojekts.

56 Albrecht (Fn. 50), Art. 19a N 27; Fingerhuth/Schlegel/Jucker (Fn. 12), Art. 19a N 9; HugBeeli (Fn. 13), Art. 19a N 253.

57 Botschaft zu einem Bundesbeschluss über die ärztliche Verschreibung von Heroin vom 18. Februar 1998, BBl 1998 II 1607 (zit. Botschaft Heroinverschreibung), 1610. verbot von sogenannten verbotenen $\mathrm{Be}$ täubungsmitteln ausgegangen wurde. 58

40 Zusammenfassend halte ich fest, dass das Gesuch der Universität Bern um Abgabe von Cannabis basierend auf der bestehenden gesetzlichen Grundlage grundsätzlich bewilligungsfähig ist.59 Sofern die Bewilligungsvoraussetzungen erfüllt sind, besteht auch ein Rechtsanspruch auf Erteilung der Bewilligung.

\section{Experimentartikel}

41 Aktuell liegen Art. 8a E-BetmG und die dazugehörige Pilotverordnung im Entwurf vor.60 Unabhängig vom Wortlaut der beiden geplanten Erlasse zeigt sich anhand der obigen Ausführungen, dass damit ein Problem adressiert wird, das nicht existiert. Dies ist deshalb problematisch, weil der Gesetzesvorschlag neben der bereits bestehenden Konstruktion der Strafbarkeit des unbefugten Konsums - eine weitere gesetzessystematische Anomalie darstellt. Dass dem so ist, zeigen die folgenden Überlegungen zu Art. 8a Abs. 2 E-BetmG, der festlegt, von welchen Bestimmungen des BetmG im Fall eines Pilotprojekts abgewichen werden kann:

«Der Bundesrat regelt die Voraussetzungen für die Durchführung von Pi-

58 Botschaft Heroinverschreibung (Fn. 57), 1610.

59 Mit gleichem Ergebnis aber alternativer Begründung: Franziska Slongo, Rechtsgutachten Pilotversuch Cannabiskonsum zu Genusszwecken, in: Medienmitteilung des Gemeinderats der Stadt Bern vom 14. März 2016, «Cannabisregulierung: Uni erhält Auftrag für Forschungsprojekt»; ebenso Samuel Schweizer, Die kontrollierte Abgabe von Cannabis als wissenschaftliche Forschung aus rechtlicher Sicht, in: sui-generis 2018, S. 205.

60 Weiterführend: Valérie Junod, Cannabis utilisé à des fins non-médicales : feu vert pour les essais pilotes ciblés?, in: sui-generis 2018, S. 414. 
lotversuchen. Dabei kann er von den Artikeln 8 Absätze 1 Buchstabe d und $5,11,13,19$ Absatz 1 Buchstabe f und 20 Absatz 1 Buchstaben d und e abweichen.»

\section{Abweichung von Verkehrsverbot und Ausnahmesituationen}

42 Gemäss vorliegendem Gesetzesvorschlag soll vom Verbot betreffend Anbau, Einfuhr, Herstellung und Inverkehrbringen von Cannabis (Art. 8 Abs. 1 lit. d BetmG) ebenso wie von den gesetzlich definierten Ausnahmesituationen (Art. 8 Abs. 5 BetmG), abgewichen werden können. Das ist nicht schlüssig, da es gerade dieser Artikel ist, der den Umgang mit Cannabis im Rahmen von wissenschaftlicher Forschung bereits regelt.

\section{Abweichung von}

\section{Verhaltensvorschriften}

43 Weiter soll abgewichen werden können von den Verhaltensvorschriften für Ärzte und Tierärzte. Gemäss Art. 11 BetmG sind Ärzte und Tierärzte verpflichtet, Betäubungsmittel nur in dem Umfang zu verwenden, abzugeben und zu verordnen, wie dies nach den anerkannten Regeln der medizinischen Wissenschaften notwendig ist. ${ }^{61}$ Hierbei handelt es sich um Verhaltensvorschriften, die im Kontext der Befreiung von der Bewilligungspflicht zu verstehen sind.62 Art. 9 BetmG legt fest, dass die bewilligungspflichtigen

$6 1 \longdiv { \text { Die Verwendung bezeichnet die «Zuführung von } }$ Betäubungsmitteln in den Körper des Patienten ohne dessen aktives Mitwirken» (Fingerhuth/Schlegel/Jucker [Fn. 12], Art. 9 N 2). Die Verschreibung ist das «Ausstellen eines Rezeptes für Patientinnen und Patienten oder Tierhalterinnen und Tierhalten, damit diese Arzneimittel mit kontrollierten Substanzen beziehen dürfen» (Art. 2 lit. g BetmKV).

62 Fingerhuth/Schlegel /Jucker (Fn. 12), Art. 11 N 2.
Handlungen (beziehen, lagern, verwenden, abgeben) von Medizinalpersonen ohne Bewilligung vorgenommen werden dürfen. Die Bewilligungsbefreiung gilt, sofern sich die Medizinalperson an die Verhaltensvorschrift hält. Tut sie das nicht, benötigt auch sie eine Bewilligung.

44 Eine weitere Abweichung betrifft die Vorschrift, dass Apotheken Betäubungsmittel nur auf ärztliche oder tierärztliche Verordnung hin abgeben dürfen (Art. 13 BetmG). Diese Norm wurde eingeführt, um «die das Publikum interessierende Bezugsvorschrift ausdrücklich zu wiederholen».63 Sie hat insofern keinen selbständigen Charakter, sondern wiederholt die die Allgemeinheit interessierende Standardsituation. Für die Ausnahmesituation nach Art. 8 Abs. 5 BetmG, welche die Allgemeinheit nicht per se betrifft, ist die Norm darum nicht einschlägig. Sofern Apotheken eine Bewilligung für die Abgabe von Cannabis erhalten können, muss es auch aus rationalen Überlegungen möglich sein, dass Art. 13 BetmG nicht zur Anwendung gelangt. Andernfalls könnte die Abgabe durch jede andere Institution, die über eine Bewilligung nach Art. 8 Abs. 5 BetmG verfügt, stattfinden, denn für diese gälte die Verhaltensvorschrift ohnehin nicht.

45 Zusammenfassend zeigt sich, dass im Rahmen von wissenschaftlicher Forschung nach geltendem Recht eine Bewilligung für die Abgabe von Cannabis erteilt werden kann. Die Verhaltensregeln von Art. 11 und 13 BetmG sind in diesem Fall nicht mehr massgeblich, weshalb es keine Notwendigkeit gibt, von ihnen abzuweichen.

63 Botschaft BetmG 1951 (Fn. 23), 856. 


\section{Abweichung von Sanktionsnormen}

46 Strafbar macht sich unter anderem, wer öffentlich zum Betäubungsmittelkonsum auffordert oder öffentlich eine Gelegenheit zum Erwerb oder Konsum von Betäubungsmitteln bekannt gibt (Art. 19 Abs. 1 lit. f BetmG), ebenso wie wer als Medizinalperson Betäubungsmittel anders als nach Art. 11 oder 13 BetmG verwendet oder abgibt bzw. wer als Arzt oder Tierarzt Betäubungsmittel anders als nach Art. 11 BetmG verschreibt (Art. 20 Abs. 1 lit. d und e BetmG).

Wie beim Konsum werden damit Handlungen mit Strafe bedroht, die gar nicht bewilligungspflichtig sind. Die hier genannten Fälle verdeutlichen die unerwünschten Konsequenzen, die sich aus Strafnormen ergeben können, die in Abweichung des Normkonzepts formuliert werden. In casu rächt sich das, weil die Strafnormen dazu führen, dass eine Handlung trotz Vorliegen der möglichen Bewilligungen mit Strafe bedroht wird. Besonders illustrativ ist das Beispiel eines Arztes, der sich, wenn er im Rahmen einer wissenschaftlichen Studie Cannabis abgibt, strafbar macht, obwohl er sogar über eine Bewilligung hierfür verfügt, wobei jede Person anderen Berufs in derselben Situation straffrei bliebe. Das kann insofern vernachlässigt bleiben, als dass die beiden Strafnormen aufgrund eines gesetzlichen Rechtfertigungsgrundes im vorliegenden Fall keinen Bestand haben dürften. Als Folge davon ist eine Abweichung von den genannten Strafnormen nicht notwendig.

\section{Fazit}

48 Die obigen Ausführungen haben gezeigt, dass die Auslegung der betäubungsmittelrechtlichen Normen bereits heute - ohne Experimentartikel - äusserst komplex ist. Die Gründe dafür sind im teilweise inkonsistenten Regulierungskonzept und in der uneinheitlichen Terminologie zu finden. Die daraus resultierende Rechtsunsicherheit ist erheblich und kann mitunter zu irreführenden Verallgemeinerungen führen, wie beispielsweise der Konsum von Cannabis sei verboten. Da ein solches generelles Verbot gerade nicht besteht, kann es keinen Anlass zur Einführung eines Experimentartikels geben. Sollten andere Bestimmungen einer Abgabe von verbotenen Betäubungsmitteln zum Konsum im Rahmen von wissenschaftlicher Forschung im Weg stehen, wären diese konkret zu benennen und anzupassen. Der aktuelle Gesetzesvorschlag führt jedoch systematisch zu einem Konflikt, mit dem sich das BetmG weiter von einem konsistenten Regulierungskonzept entfernt. Darum wird der vorliegende Regulierungsvorschlag, sollte er so angenommen werden, zu weiteren Auslegungsschwierigkeiten führen und der Verständlichkeit des Gesetzes abträglich sein, was wiederum eine für die Behörden, wie auch für die Bürgerinnen und Bürger vorhersehbare Rechtsanwendung erschwert.64 All dies deutet darauf hin, dass momentan nicht primär der Konsum von Cannabis, sondern die Auslegung und Weiterentwicklung der Rechtsgrundlagen das eigentliche Experiment sind. Eine Anpassung derselben wäre darum wünschenswert und zwar in Umsetzung eines stringenten Regulierungskonzepts.

64 Vgl. zum Normkonzept im Allgemeinen: Gesetzgebungsleitfaden des Bundes, 3. Aufl. Bern 2007, S. 353 . 